\title{
Linking the lonely: an exploration of a communication technology designed to support social interaction among older adults
}

\author{
Chiara Garattini · Joseph Wherton • \\ David Prendergast
}

Published online: 7 June 2011

(C) Springer-Verlag 2011

\begin{abstract}
This article explores mixed methods data gathered from a pilot of a communication system prototype in the homes of 19 older adults over a period of 10 weeks. The system has been designed to enhance communication among both friends and strangers and has been developed as a possible tool to increase interaction in older adults suffering from social isolation and loneliness. The paper uses remote logging data to inform discussion of how such a technology was received and utilised over time in a home setting. Qualitative data gathered via entry and exit interviews, and weekly checkpoint calls were used to provide deeper insight into patterns and practices identified via the logs.
\end{abstract}

Keywords Information and communication technology · Ageing $\cdot$ Loneliness

C. Garattini $(\bowtie) \cdot J$. Wherton $\cdot$ D. Prendergast

Technology Research for Independent Living (TRIL) Centre,

Dublin, Ireland

e-mail: chiara.garattini@ trilcentre.org

J. Wherton

e-mail: whertonj@tcd.ie

D. Prendergast

e-mail: david.k.prendergast@intel.com

C. Garattini

School of Public Health, Physiotherapy and Population Science,

University College Dublin, Dublin, Ireland

J. Wherton

Trinity College Institute of Neuroscience,

Trinity College Dublin, Dublin, Ireland

D. Prendergast

Intel Labs, Health Research and Innovation, Leixlip, Ireland

\section{Social isolation, health and technology}

Loneliness and social isolation are a high priority problem for ageing populations around the world. Loneliness and social isolation are often a consequence of mobility constraints, bereavement, declining social networks or physical relocation and have been proven to be closely associated with poor mental and physical health outcomes. International studies have estimated that between 5 and $16 \%$ of older adults experience loneliness [1,2]. Decline in mental and physical health influence and are influenced by loneliness. For example, restricted physical mobility reduces engagement in social activities, which can further lead to depression or loss of appetite [3], increasing the chances of rapid decline in health condition. Contact with family and friends is important for perceived social support $[4,5]$, and participation in social events plays a role reducing risks of depression, anxiety and cognitive impairment [6].

Voluntary sector befriending schemes have been found to be effective in reducing loneliness. Monk and Reed [7] describe a befriending scheme in London that uses telephone conference calls and weekly one-to-one calls to connect older adults over the phone. They found that the users perceived an intrinsic benefit in actively engaging, or listening to a conversation. Regular contact also provided reassurance and instrumental gains through information sharing. King et al. [8] report the benefits of telephone reassurance service for older adults living at home. Initially, the scheme was set up for emergencies, but was later found to play an important role in providing a sense of security and well-being.

A number of studies have also demonstrated the potential role of internet communication technology in reducing loneliness and improve quality of life among older adults at risk of isolation. White et al. [9] explored 
the effect of internet use in a retirement community and demonstrated through assessments that reconnecting social ties decreased level of loneliness. Similarly, Groves and Slack [10] explored the impact of a computer-training programme with 20 nursing home residents. Pre- and postevaluations showed an increase in independence and engagement in social activities.

Internet communication technologies offer individuals new ways to help remain connected with peers and family members in a flexible and inexpensive way. However, technologies of this type are often inaccessible to the older section of the population due to decline in cognitive, sensory and physical abilities. Furthermore, many older adults, especially those in the late stages of the life course, are not regular computer users and would experience problems with interface conventions (e.g. scroll bar), terminology (e.g. 'File'), and operating system (e.g. clicking mouse correctly) [11, 12].

Numerous studies have explored the design requirements for email systems that can be easily used by older adults with little or no computer experience [11, 13, 14]. Czaja et al. [13] developed a simple messaging system to support social connectedness among 36 women between the ages of 55 and 95 with little computer experience. The trial lasted for 6 months, and it was found that the users could work the system with a minimal amount of difficulty, and that they found it useful. Importantly, participants indicated that the system facilitated social interaction and provided a chance to meet new people. However, a significant decline in use over time was also found. This was attributed to the fact that people were busy and the system had limited functionality. This seems to indicate that although the system was easy to use, it needed to be perceived as useful in order for it to be adopted in the long term. The system would need to be expanded without unnecessarily increasing its complexity.

\section{Purpose of the study}

During summer 2009, the Building Bridges system (see Sect. 3) was deployed in a home trial with 19 older adults (primary participants) for 10 weeks. Participants included a diverse range of older adults with considerable variation in levels of social connectivity and experience with technology. In addition, 17 friends and family members (secondary participants) were nominated at the discretion of the participants to use a client version of Building Bridges; this CD version can be quickly installed on a standard PC and allowed secondary participants to also participate within the network.

The study was designed to obtain an insight in how the system could be used in a real-world setting (i.e. outside of laboratory and into the home) and explore how such a system impacted on participants' social connectedness. The aim was to deploy technology in the home as less intrusively as possible. Unlike most communication technologies, this system is designed to provide opportunistic social interaction in a non-intrusive way to encourage social interaction among strangers. Through analysis of system usage and users' experiences, the study reports how the system was used by primary participants. Implications for designing recreational technology to support social connectivity in order to reduce risks of isolation are also discussed.

\section{The building bridges system}

Drawing from previous research around loneliness among older adults, the Technology Research for Independent Living (TRIL) Centre developed a device as part of the Building Bridges project to encourage peer-to-peer social engagement among older adults. The device consists of a 12-inch touch screen computer in a custom-made stand, a phone handset with functioning cradle and speakers (see Fig. 1). The software uses VoIP with a customised Flashbased interface developed with participation from older adults that allows users to interact with four main features. These features are briefly described here (see [15] for more details about the system and design process).

\subsection{Broadcast and chat}

Users can listen to regular broadcasts (e.g. news, documentaries, health lectures, stories and music). A guide to the broadcasts (time, day and topic) can be viewed on the left of the Main Menu screen. At the scheduled time, a message appears on the screen inviting the user to listen to the broadcast. If they wish to listen, they press a button on the screen. During the broadcast, icons that represent other people (i.e. avatars; see Fig. 2) who joined are shown on

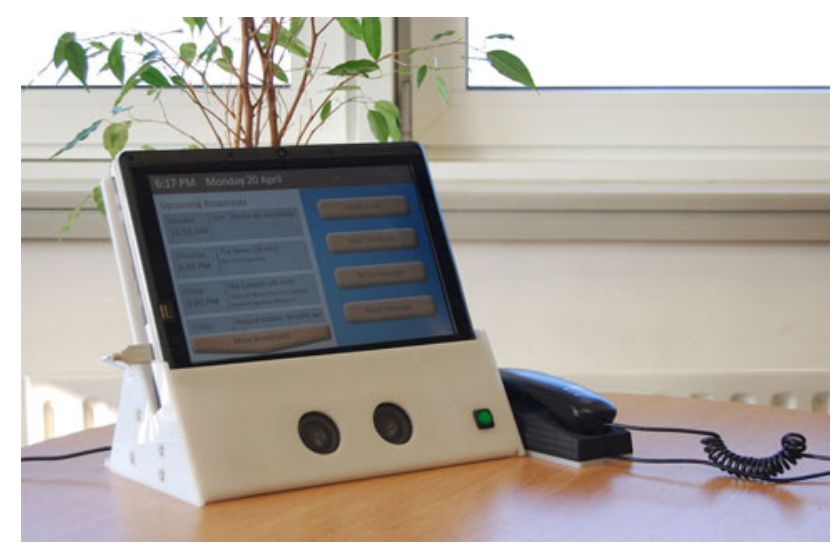

Fig. 1 The building bridges device displaying the Main Menu screen 


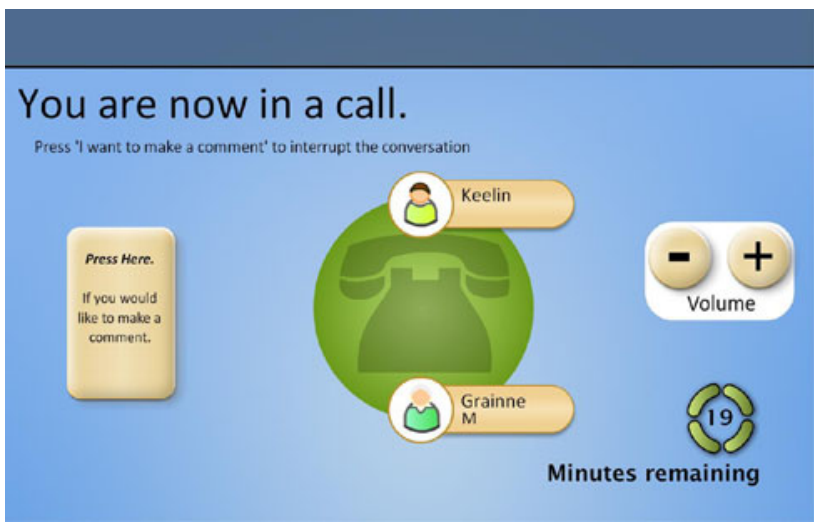

Fig. 2 The screen display during a group chat

the right of the screen. When the broadcast is over, the user can join a 'group chat' with the other listeners by lifting the phone handset. During the group chat, the screen displays visual cues to support the conversation (e.g. who has entered/left the call, if someone wishes to interrupt the conversation and a counter representing how much time is left on the group chat).

\subsection{Calls}

Users can make calls to one or more people. To start a call, the user presses the button labelled 'Make a Call' on the main menu screen. This leads to another screen showing a list of other people they can call. They then select the person(s) they wish to call. If the person they wish to call is not available (i.e. their device is switched off), the icon is shown as grey and a red cross appears in front of it when pressed. Once all contacts are selected, the user presses the button labelled 'Call'. During the call, the user is provided with the same display as the after-broadcast chat without the counter. Figure 2 presents an image of the group chat display.

\subsection{Messaging}

Users can write messages that are up to 160 characters long. To write a message, the user first presses the button labelled 'Write message'. This leads to a screen presenting a touch screen keyboard. The letters are arranged alphabetically on the keyboard. If the user wants to use numbers or punctuation in the message, they are required to press a button that changes the keyboard mode. Similarly, if they wish to use capitals, a separate button is used to change the mode from lower- to upper-case.

\subsection{Tea room}

The Tea Room is an audio chat room that users can access anytime day or night. The user enters this by pressing the button labelled 'Visit Tea Room' on the main menu screen. If another person entered, their icon would show up on the screen. Users needed to lift the handset to talk, but they can also listen to ongoing conversations through the speakers. During the trial, classical music was played when the room did not include any other people. A series of background pictures from web cameras positioned around Dublin were also displayed on the screen.

\section{Method}

\subsection{Sample}

This study included two sample sets. The primary participants were 19 older adults who were provided with broadband connectivity and a Building Bridges device. The secondary sample included their friends and family members who volunteered to use a PC client version of the software on their own computers. This allowed the primary participants to contact their own 'friends and family' via the system, as well as the other primary participants. This paper focuses on the data from the older adult users (primary sample); secondary participants' engagement with the system is taken into account only when they were recipients of calls and messages from primary participants.

Primary sample There were 19 primary participants (7 male, 12 female) aged between 65 and 84 and were recruited through the Technology Research for Independent Living (TRIL) research clinic at St James's Hospital in Dublin. The TRIL Clinic offers a comprehensive geriatric assessment to community-dwelling people over 60 years of age in Ireland. Assessments include physical health, cognition, mood and social connectedness. All participants lived in their own homes in Dublin or surrounding areas. Of these, $47 \%$ lived alone and the remaining 53\% lived with a spouse. The majority did not own a computer (68\%). According to the DeJong Loneliness Scale [16], 6 (32\%) of the participants were classified as 'socially' lonely and $5(26 \%)$ as 'emotionally' lonely. Loneliness is a subjective state of negative feeling associated with perceived social isolation. Whereas social loneliness stems from a perceived lack of social contacts or engaging social network, emotional loneliness is perceived as arising from the absence of an intimate relationship or close attachment.

It has been suggested that social loneliness can be associated with a lack of social integration, and emotional loneliness is the result of psychological factors [17]. Table 1 lists the 19 participants and background characteristics as identified through the TRIL clinic. As the study is exploratory, a broad range of participants were included 
Table 1 Summary of primary participant profiles (names have been changed)

\begin{tabular}{|c|c|c|c|c|c|c|}
\hline Participants & Age & Lives alone & Social lonely & Emotional lonely & Use a computer & PC client contacts \\
\hline Alice & 69 & & & & $\mathrm{x}$ & 2 \\
\hline Bert & 78 & $\mathrm{x}$ & & & $\mathrm{x}$ & - \\
\hline Deidre & 67 & & & $\mathrm{x}$ & & - \\
\hline Eamon & 74 & & & & & 1 \\
\hline Emer & 65 & $\mathrm{x}$ & & & $\mathrm{x}$ & 1 \\
\hline Grace & 75 & $\mathrm{x}$ & $\mathrm{x}$ & & $\mathrm{x}$ & 1 \\
\hline Grainne & 74 & & $\mathrm{x}$ & $\mathrm{x}$ & & 1 \\
\hline Joan & 75 & $\mathrm{x}$ & $\mathrm{x}$ & & & 2 \\
\hline Julia & 76 & & $\mathrm{x}$ & $\mathrm{x}$ & & 2 \\
\hline Karen & 69 & & & & & 3 \\
\hline Kevin & 84 & $\mathrm{x}$ & & & & 1 \\
\hline Liam & 70 & & & & & 1 \\
\hline Louise & 74 & $\mathrm{x}$ & $\mathrm{x}$ & $\mathrm{x}$ & & - \\
\hline Martha & 72 & $\mathrm{x}$ & & & & - \\
\hline Patrick & 88 & $\mathrm{x}$ & $\mathrm{x}$ & & $\mathrm{x}$ & - \\
\hline Sean & 65 & & & & & - \\
\hline Sue & 76 & & & & & 1 \\
\hline Theresa & 81 & $\mathrm{x}$ & & $\mathrm{x}$ & & 1 \\
\hline Tim & 75 & & & & $\mathrm{x}$ & - \\
\hline
\end{tabular}

in relation to age, loneliness and experience with computers.

Friends and family The 19 primary participants were asked to nominate family and friends to use the PC client version of the software. Nominees that agreed to take part were provided with the software, a user guide for installing the software and USB phone handsets. The PC client allowed them to make calls and messages the participant that nominated them. They could also access the Tea Room. However, they did not have access to the broadcast feature as this was exclusively meant for the 19 primary participants. There were 17 PC client users in total. Eleven of the primary participants had at least one PC client contact (see Table 1).

\subsection{Ethics}

Participants were required to provide informed consent before taking part. All were provided with an information sheet explaining the purpose of the study, the data being collected and how long they would have the technology. All participants were provided with a contact telephone number if they needed any assistance with the device. They could also contact the researcher via the system itself using the calls or messaging features. It was emphasised to all participants that they could use the system as much or as little as they liked and should not feel obliged to use the device at anytime. Participants were not provided with personal information or contact details of the other participants. All names presented in this paper are pseudonyms.

\subsection{Procedure and support}

Each primary participant was given the Building Bridges device for 10 weeks. They were told to use it as much, or as little, as they liked. The device allowed participants to use all four features described in Sect. 2. Each primary participant could contact the other primary participants through the system, as well as their respective PC client users. The trial included four separate home visits and one focus group with all participants a month after the conclusion of the home deployment:

Visit 1 ( 1 h)_Recruitment Researchers visited the participants' homes to explain the purpose of the project and describe the device. Participants were also asked to identify any friends or family members who may be interested in receiving the $\mathrm{PC}$ client version of the software. An information sheet was left with the participants, along with information leaflets for any friends and family who wished to be involved.

Visit $2(1 \mathrm{~h})$-Entry interview The interview took part in participants' homes and included a semi-structured question script, focusing on health, social routines, changes to social network and experience with technology.

Visit 3 ( 1 h)_Deployment and Training The device was installed in homes in a location of a participant's choosing (e.g. spare room, lounge or kitchen) by the researcher. Each 
participant was shown a short video, lasting $6 \mathrm{~min}$, which described the system. They could access the video at anytime during the trial by pressing a button on the touch screen. The researcher then demonstrated each feature to the participant and showed how to turn the device on/off.

Visit $4(1 h)$-Exit interview At the end of the trial, the researchers removed the device and conducted an exit interview with the participant. This focused on the user experience with the system and perceived impact on their social connectedness. In addition, the researchers carried out a system questionnaire with participants. The device was then removed from the home.

Focus group ( 1 h) One month after the trial had concluded, the 19 primary participants were invited to a focus group and lunch. It provided an opportunity for the participants to formally meet each other in person for the first time, exchange contact details if desired and discuss their experiences with the system along with their views about how it could be further customised or improved.

The participants were able to contact the research team at anytime during the trial. This could be done via the messaging feature by sending a message to a TRIL Help contact. A contact phone number was also left with all participants. In addition, weekly calls were made to each primary participant to report any technical issues, such as system breakdowns. For continuity, the participants interacted with an assigned researcher throughout the trial.

\subsection{Data collection and analysis}

Use of the system was logged remotely for each primary participant. This included the time, frequency and duration that each feature was used. The participants were aware their usage was being logged. A repeated measures ANOVA was used to test the main effect of time over the 10 weeks. The Greenhouse-Geisser [18] three-step approach to significance testing was employed when relevant. In this case, the uncorrected degrees of freedom, the corrected $p$ value and the epsilon value of the correction factor are reported. Post hoc $t$ tests were calculated to compare use between the first half (Time 1, weeks 1-5) and second half (Time 2, weeks 6-10) of the trial. The relationship between system use and background characteristics, including gender, experience with computers, living alone and loneliness, was also explored using $t$ tests. However, it is worth noting the limitations due to the small sample size ( $n=19$ primary participants).

Exit interviews and focus group were audio recorded and analysed to identify common themes occurring across the different cases. Themes emerged through a collective analysis of data in which researchers presented case notes from their respective participants to the rest of the research team. During these presentations key issues and insights were captured via post-it notes. Once all cases had been presented, the post-it notes were grouped and categorised on a white board to highlight themes.

\section{Results}

\subsection{System use}

\subsubsection{Broadcasts}

There were 125 broadcasts in total (2-3 per day), played between $11 \mathrm{am}$ and $8 \mathrm{pm}$ everyday (excluding weekends). Table 2 summarises broadcast types. Length ranged from 6 to $30 \mathrm{~min}$. There were five main broadcast types: News (which was played at 11 am every week day), a soap opera ('The Archers'), documentaries (e.g. 'History of Ireland', 'Walks around Ireland'), health (e.g. 'Coping with Falls' and 'Helping your Memory') and entertainment (e.g. comedy and music). Nearly all broadcasts were obtained from internet sources. Six of the health broadcasts were created by TRIL research team.

Overall, $104(84 \%)$ of all broadcasts were attended by at least one person. Overall, $74(59 \%)$ of broadcasts were attended by two or more people (opportunities to talk) and $28(22 \%)$ of all broadcasts led to a group chat (decision to talk). Health broadcasts showed the highest rate of attendance of two or more people $(84 \%)$ and highest rate of chats $(58 \%)$. The broadcast type least likely to lead to a chat was the soap opera $(6 \%)$, followed by the news broadcasts $(10 \%)$. Just over half $(54 \%)$ of post-broadcast chats were between 2 people, $25 \%$ included three people, and $21 \%$ include $4-5$ people. The average duration for post-broadcast chats was $4 \mathrm{~m} 44 \mathrm{~s}$. On average, health broadcasts led to the longest chats $(6 \mathrm{~m} 30 \mathrm{~s})$. One chat lasted the full $20 \mathrm{~min}$, which followed a health broadcast on 'brain fitness'. The shortest post-broadcast chat followed the soap opera and lasted just $9 \mathrm{~s}$.

Figure 3 shows the number of broadcasts attended by two or more people and frequency of broadcast chats over the 10 weeks. There was a significant main affect of time on broadcast attendance over the 10 weeks, $F(9,126)=5.5$, $\varepsilon=.4, p<.01$. However, a repeated measures t-test did not show a significant change in attendance rate between the first half (weeks 1-5) and the second half (weeks 6-10). With regard to post-broadcast chats, there was a significant change in attendance observed over time, $F(9,126)=3.5$, $\varepsilon=0.3, p<.05$. Post hoc analysis revealed a significant drop in attendance to post-broadcast chats between the first half (weeks 1-5) and the second half (weeks 6-10), $t(18)=2.7, p<.05$. Attendance to broadcast was significantly higher for female participants than male participants, $t(17)=2.3, p<.05$. Female participants also attended 
Table 2 Summary of broadcast types and attendance

\begin{tabular}{llllll}
\hline Broadcast type & No. of broadcasts & \% Joined (at least 1) & \% Joined $>1$ & \% Led to chat & Average chat length \\
\hline News & 51 & 78 & 49 & 10 & $3 \mathrm{~m} 53 \mathrm{~s}$ \\
Soaps & 16 & 69 & 31 & 70 & 6 \\
Documentary & 30 & 90 & 84 & 22 & $3 \mathrm{~m} \mathrm{~s} 5 \mathrm{~s}$ \\
Health & 19 & 95 & 78 & 58 & $6 \mathrm{~m} 30 \mathrm{~s}$ \\
Entertainment & 9 & 100 & 44 & $5 \mathrm{~m} \mathrm{~s}$ \\
\hline
\end{tabular}

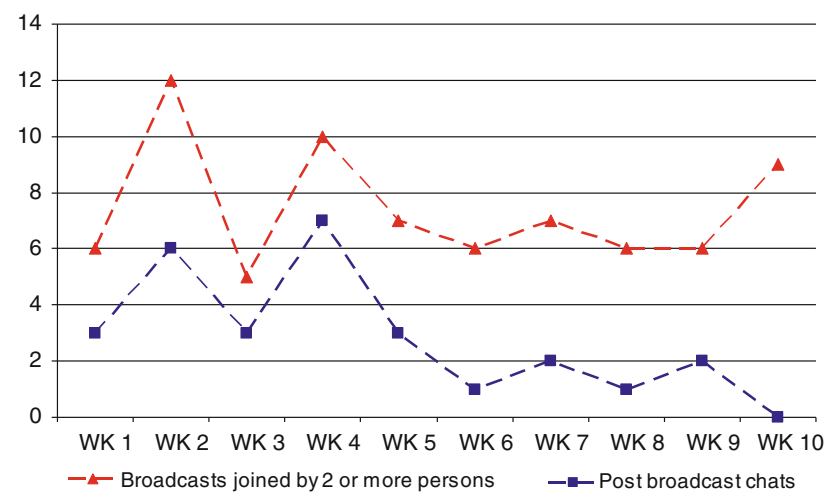

Fig. 3 Frequency of broadcast joined by 2 or more people and postbroadcast chats across 10 weeks

more post-broadcast chats, $t(17)=2.2, p<.05$. Attendance to post-broadcast chats was also related to loneliness, in which those identified as emotionally lonely attended more frequently than those who were not. Social loneliness, living alone and experience with computers were not found to have a significant relationship with this feature.

Participants reported the opportunistic nature of talking to other people after a broadcast; this would often include people who they would not have otherwise thought to contact. One male participant likened it to a social encounter in a public place: 'It was the typical lamp post at the corner of the street and three or four chatting' (Kevin). Some who were reluctant to initiate a call also considered it to be the main opportunity to chat: 'I was too shy to make a call... I would talk to people after the broadcast' (Louise). The shared experience of the broadcast also provided a topic of conversation even for those participants that were less active in calls and messages (e.g. men): '[we were] talking about the broadcast because that was the common bond. And you had the men join in then' (Louise). These accounts indicate that broadcasts fulfilled their design purpose in somewhat encouraging social interaction by the provision of an opportunistic discussion topic. However, as the chats were not facilitated, some reported difficulties in following the conversation or breaking into it. Some participants joined the post-broadcast chat in moments when participants were talking about intimate issues (or at least

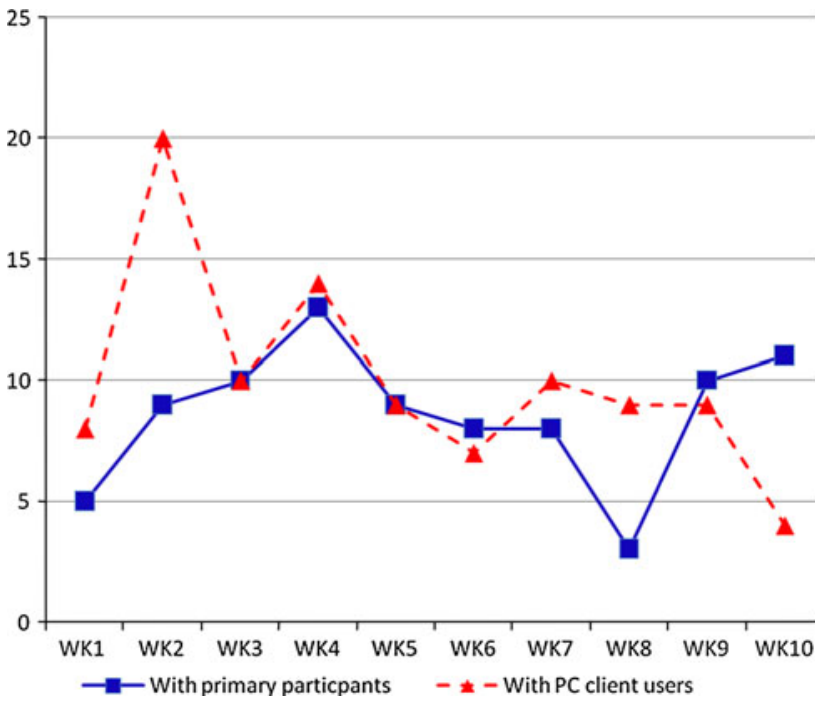

Fig. 4 Total conversations (calls answered) initiated by 19 primary participants to other primary participants and PC client users

what was perceived as intimate by the person joining) and felt disinclined to reengage.

\subsubsection{Calls}

In total, 361 calls were attempted by the primary participants. Of these, 187 were answered by the recipient. Out of the conversations that took place, $47 \%$ were between primary participants (87 total calls initiated and answered), and 53\% were between primary participants and their respective PC client nominees (100 total calls initiated and answered). Any calls made to TRIL Help were excluded from the analysis. Figure 4 shows frequency of answered calls across the 10 weeks. There was no significant change in use of this feature observed across the 10 weeks.

Figure 5 shows the average duration of calls over the 10 weeks. Average call duration between primary participants was $8 \mathrm{~min} 40 \mathrm{~s}$. There appeared to be an increase in call duration. For example, week 1 showed the shortest average call duration ( $4 \mathrm{~m} 36 \mathrm{~s}$ ), and week 10 showed the longest average call duration ( $13 \mathrm{~m} 12 \mathrm{~s})$. However, no significant change in time spent using this feature was observed over the 10 weeks. Similarly, call duration with 


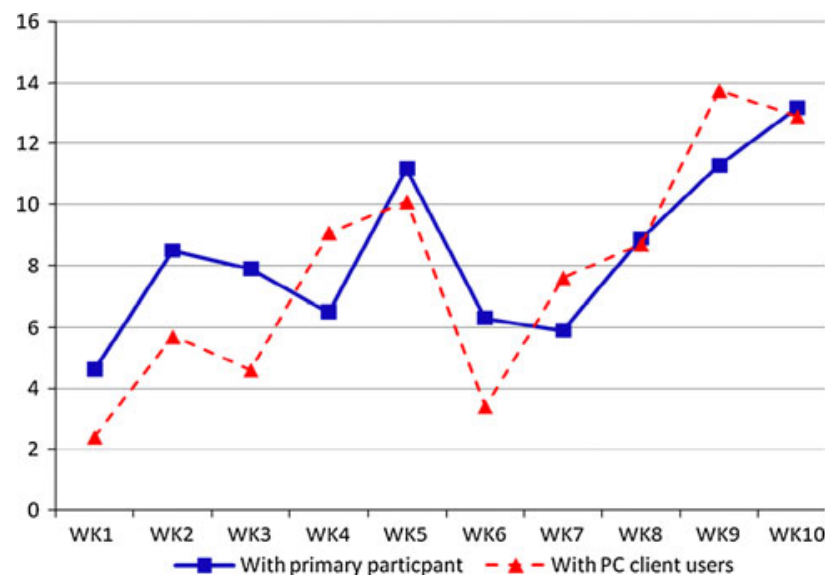

Fig. 5 Mean duration (min) of conversations over 10 weeks

PC client users peaked towards the end of the trial, the highest average being in week $9(13 \mathrm{~m} 54 \mathrm{~s})$ and the lowest in week $1(2 \mathrm{~m} 24 \mathrm{~s})$, but no significant change in duration was observed.

Analysis of participant background characteristics revealed that females had significantly more calls with other primary participants than males, $t(17)=2.3$, $p<.05$. Loneliness was also associated with this feature, since those identified as socially lonely had more calls with other primary participants, $t(17)=-2.4, p<.05$. Living status and experience with computers were not found to be significant factors in using this feature.

The majority of calls between primary participants were one-to-one conversations. Only 7 group calls took place over the 10 weeks outside the broadcast chats and Tea Room conversations. It appeared that the low frequency of group calls was partly related to lack of awareness about this feature: 'I didn't know you could make a group call, that would have made a difference!'(Kevin). Participants generally associated the group chat feature with the broadcasts or the Tea Room, and many did not register that they could initiate their own multi-person calls. This was despite the fact that group call procedure was the same as that used for sending a group messages and that this functionality was also shown during the deployment and training visit and was included in the introduction video.

\subsubsection{Messages}

In total, 574 messages were sent by the primary participants. Fifty-two messages (9\%) were group messages. For the purposes of analysis, group messages were counted as one message. The majority (79\%) of messages sent were to other primary participants, the remaining $21 \%$ were sent to PC client users (family and friends). Any messages sent to TRIL Help were excluded from the analysis. Figure 4

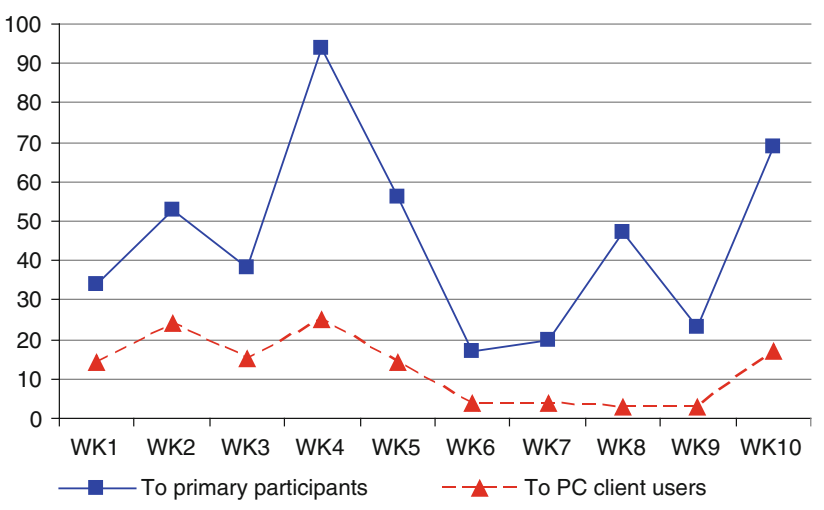

Fig. 6 Messages sent by 19 primary participants to other participants and PC client users across 10 weeks

shows the frequency of messages sent to other primary participants and PC client users across the 10 weeks.

Figure 6 shows frequency of messages sent over 10 weeks. On average, 45 messages were sent to other primary participants per week. This ranged from 17 (week 6) to 94 (week 4). There was a significant main effect of time on frequency of messages sent to other primary participants, $F(9,126)=3.4, \varepsilon=.5, p<.05$. Repeated-measures t-test showed a significant drop in messages sent to other primary participants from the first half (week 1-5) to the second half (week 6-10) of the trial, $t(18)=2.1, p<.05$.

On average, 12 messages were sent to PC client users per week. There was also a significant main effect of time on frequency of messages sent to PC client users, $F$ ( 9 , $63)=3.6, \varepsilon=.3, p<.05$. However, the difference in messages sent between the first half (weeks 1-5) and second half (week 6-10) was not found to be significant. Background characteristics associated with use of messaging included gender, in which females sent significantly more messages than males, $t(17)=3.6, p<.05$ and loneliness, in which those who were socially lonely sent significantly more messages than those who were not, $t(17)=-2.2$, $p<.05$. Living status and experience with computers were not significant factors in frequency of messages sent.

According to the participants, the messaging had the dual purpose of lightweight social interaction (e.g. greeting or acknowledgement) and an instrumental role for arranging a call or meet up in the Tea Room, as well as outside of the system (e.g. meeting in a local park): 'Last Saturday a message said these women were walking in the park and I would have been interested in that, I would have done that' (Karen). Some of the comments seem to highlight an increasing intimacy of the message content through time: 'It got more friendly and more personal, how are you and mind yourself' (Louise).

For simplicity, the word limit was restricted to the writing space on the screen, which means that up to 160 
characters could be used in one message (more like a text than an email). This constraint allowed for recommended font size for the user group (Arial 14) and avoided added complexity to view text beyond the writing space, such as scroll bars. However, utilising this constraint enforced unexpected limitations for the users. Many wanted to still write longer letters in conventional form, rather than the short hand 'text language'. They also wanted to maintain standard letter openings (e.g. 'Dear...') and endings (e.g. 'Regards...'), as would be used for letters or postcards. Consequently, the space allowed in the writing space was not sufficient for the participants. Tendency to avoid abbreviated language also added an extra step of planning before writing the messages: 'You had to think "what can I say"... It would have to be short' (Theresa).

\subsubsection{Tea room}

On average the participants entered the Tea Room 40 times over the course of the trial. There was a large range across participants. For example, Grace made 196 entries in total, spending just over $81 \mathrm{~h} 18 \mathrm{~min}$ in the Tea Room. In contrast, Bert spent $3 \mathrm{~min} 36 \mathrm{~s}$ in total across 10 entries. Unlike the other device features, the Tea Room is a state of the device as opposed to an event, and therefore, the use of this feature does not necessarily reflect social interaction. For example, a user may keep the Tea Room application open but leave the house; therefore, time duration of use is not reliable for assessment of social interaction. At the same time, the number of accesses throughout the day was also not a reliable indicator of social interaction as some participants liked the music and stayed alone in the Tea Room to listen, while others accessed and left it many times throughout the trial to check if someone was in.

However, participants' comments about their experience of the system provided some insight into how it was used. There were quite polarised attitudes towards the Tea Room, as some people loved it while some people did not. Among some of those who did not like the Tea Room, some found it difficult to join in on a conversation. On the other hand, the Tea Room also had some great proponents. In particular, two participants, self-defined by one of them as 'late night birds' (Emer), liked to use the device in the evening and often late into the night. The Tea Room presented a very good option to see whether someone was online without calling or sending messages that they perceived as inappropriate late in the evening.

\subsection{Perceptions of the system}

An exit interview and system questionnaire was carried out to support log analysis and explore perceptions of the system. The questionnaire was a shortened version of the system questionnaire devised by Czaja et al. [13]. Table 3 summarises responses to the system questionnaire. Sixtyfour per cent of the sample reported that they were 'satisfied' or 'very satisfied' with the system as a means of communication. The messaging was ranked as the most preferred feature of communicating (47\%), followed by calls $(27 \%)$. The majority of the participants said that they liked the system because they found it 'easy to use' (76\%), liked 'meeting new people' $(65 \%)$, and liked 'communicating with others' $(65 \%)$. Forty-one per cent reported that they wanted to use the system to 'socialise with other people'. However, over half (65\%) also reported using the system as 'part of the study'. Main reasons for not using the system included being 'busy' (29\%), on 'holiday' (29\%), or 'illness' $(29 \%)$. Just under a third $(29 \%)$ reported that there was 'limited use' of the system.

\section{Discussion}

This paper has reported the usage and experience of older adults using a novel communication system designed to support social participation and connectedness. The findings show the potential for using internet-based communication technology as a means for encouraging social interaction in a flexible and unobtrusive way. The work builds on previous research showing the promise of providing group interaction over the phone and the benefits of making internet communication more accessible to older adults.

The system used in the current study was designed to provide opportunities for one-to-one and group interaction with new people. In this case, calls did not involve a facilitator. Instead, opportunistic interaction was stimulated through daily broadcasts, integrated by the calling, messaging and chat room features. This pilot study provides insight into how this new system is used in real-home settings. It provides further insight into how such technology should be developed so that it fits with what potential users need and want. The remote logs of system use, along with exit interviews, provided insight into how the system was used. It should be noted that as the study includes a small sample size, the results cannot be generalised. However, it provides further understanding into how users engage with the system and socialise through the technology.

\subsection{Engagement with the system}

When a new technology is introduced, engagement with the system may be more of a reflection of its novelty as opposed to usefulness. Other studies have previously highlighted this 'novelty effect' when piloting new 
Table 3 System Questionnaire

\begin{tabular}{|c|c|c|c|}
\hline \multicolumn{4}{|c|}{ System questionnaire $(N=17)$} \\
\hline & $(\%)$ & & $(\%)$ \\
\hline \multicolumn{2}{|l|}{ Reasons for use } & \multicolumn{2}{|l|}{ System likes } \\
\hline To socialise & 41 & Easy to use & 76 \\
\hline Learn about computers & 29 & Meeting new people & 65 \\
\hline Part of the study & 65 & Communicate with others & 65 \\
\hline \multirow[t]{3}{*}{ Fun/challenge } & 35 & Interesting/stimulating & 47 \\
\hline & & Fun & 29 \\
\hline & & Educational & 47 \\
\hline Reasons for non-use & & System dislikes & \\
\hline Bored & 6 & Hard to use & 12 \\
\hline Not enough people & 12 & Breakdowns & 41 \\
\hline Difficult to use & 0 & Problems using system & 12 \\
\hline Busy & 29 & Inconvenient & 6 \\
\hline System break downs & 18 & Intrusive & 6 \\
\hline Holiday & 29 & Limited use & 29 \\
\hline Illness & 29 & Boring & 0 \\
\hline $\begin{array}{l}\text { Satisfied using as means } \\
\text { of communication }\end{array}$ & & $\begin{array}{l}\text { Preferred feature } \\
\text { for socialising }\end{array}$ & \\
\hline Very satisfied & 35 & Broadcasts & 13 \\
\hline Satisfied & 29 & Calls & 27 \\
\hline Neutral & 18 & Messaging & 47 \\
\hline Dissatisfied & 12 & Tea Room & 13 \\
\hline Very dissatisfied & 0 & & \\
\hline
\end{tabular}

communication systems with older adults [13]. The novelty effect refers to when engagement and persistence with technology is high at the early stages simply because it is new. Usage generally peaks at the beginning and steadily declines over time.

Although a drop in use of the call feature was observed between first and second half of the trial, this change was not found to be statistically significant. The trial length (10 weeks) might have affected the 'novelty effect', and a longer study might have shown novelty effect more clearly. The lack of statistically significant drop in usage may also be partly connected with the sharp increase in use during weeks 9 and 10 for calls. This wave of increased use may be due to participants' awareness that the deployment was due to end; the perceived final phase of the trial might have been seen as the last opportunity to engage with each other.

The call feature of the system is so to speak a 'bravery check'; this is because calling someone you do not know to have a chat with requires a certain amount of courage, and most of the participants did not know each other previously to the trial. In this context, one of the objectives was to see whether people called more their friends and family (secondary participants) than the other participants (primary participants), and whether the duration of calls with former group was longer than with the latter. Another objective was to see how this behaviour changed through time, and if by the end of the trial, the data would have shown an increased engagement among participants. As the frequency of calls decreased slightly throughout the trial, their duration increased towards the end for both calls between primary participants and with friends and family. This could suggest that through time people got to know each other and had less frequent but longer and more meaningful conversations.

With regard to messaging, a significant drop was observed between the first and second half of the trial. It is likely that this feature was seen as more novel to the users than the calls. Furthermore, it provided a less intrusive from of social interaction and would have been particularly useful for initial introductions at the early stages of the trial. A novelty effect was also observed for post-broadcast chats, in which there was a significant decrease in use, despite a consistent rate of attendance to broadcasts. This means that opportunities to chat remained the same, but participants' deciding to seize on these became less frequent. The broadcasts were designed to encourage and support the meeting of new people, and so this drop would be expected once they had become acquainted. Broadcast type also influenced usage. In particular, those regarding health issues were far more likely to attract both listeners and conversation than others.

The remote logs provide useful descriptive insight into how the technology is used in a home setting. As others have highlighted, self reports alone are limited by participants' wishes to praise the researcher, rather than give an objective view [19]. The logs data were instrumental in facilitating the interview and help elicit reasons behind decisions to use, or not use, the technology. The data from remote logging also provided an overview of the extent to which users engaged with the new technology and how this changed over time. It should be noted however that with such a small sample, it is difficult to generalise the results and that participants were aware of being part of a research study and of being logged. Furthermore, a longer trial may have revealed more significant changes in usage. The nature of the trial, which aimed at being as natural and unobtrusive as possible, also meant that some confounding factors could not be controlled for. For example, some participants went away, while others could not use the system due to illness. Furthermore, a subset of the sample had additional contacts with the PC client, which may have biased their use of the device. Despite these limitations, the data provided a useful insight into how different users adopted the technology during the course of the 10 weeks trial, which could not have been established through selfreports. Relating the log data with users' background characteristics would also suggest that usage was not influenced by prior experience with the technology. 
However, gender and levels of loneliness did have some impact on system use, which is discussed in the next section.

\subsection{Socialising through technology}

The aim of the present study was to explore how the technology was used and supported social interaction in real-world settings beyond the laboratory. To this end, there was little intervention or facilitation by the researchers. It was made clear to participants at the beginning of the trial that they were free to use the system as much, or as little, as they wished. The device was located in a position established by participants (e.g. near the TV vs. upstairs in a room dedicated to mid afternoon activities); this likely had an impact on the interaction and usage with the device.

The majority of participants reported that they liked the system because it provided opportunities to meet new people and were satisfied with it as a means of communicating. However, it should also be acknowledged that over half of the participants provided being 'part of the study' as one of explanation for their engagement with the system. Despite efforts to minimise researcher effects, the duration of the trial and the awareness of being logged might have played a role in usage patterns. Nevertheless, analysis of usage data and accounts from the participants highlight a number of issues and challenges related to the development of communication technology to support social connectivity.

Firstly, there was variability in use across participants. Analysis of usage logs across features indicated that engagement differed across gender (women were more frequent users then men) and loneliness (those who showed indications of social loneliness were more regular users). This led to the problem of frequent users becoming frustrated with the relative disengagement of others. For example, there were frustrations around getting no response, or delayed responses, to messages and missed calls: 'I make contact, send messages and people don't reply' (Grace). The impact of fellow users on an individual's overall experience highlights the point that the users themselves constitute the system, not the technology alone.

Second, the system produced some expectations related to the social norms connected with usage. One of such norms for example regarded the Tea Room, the feature that obtained the most polarised feedback. At the beginning of the trial, one of the participants stayed in the Tea Room without leaving it for long periods of time while doing other things in the house; that meant that even if her avatar was in the Tea Room she was not anywhere near the device and that other participants joining in were left under the impression that someone was there even if there was nobody at the keyboard. This person was told about it by some participants, felt guilty about it and reported that she would be careful to avoid doing so again.

Third, the participants highlighted a need for greater flexibility over privacy and personal identity. During the user-centred design research done to develop the prototype, it emerged that some people had concerns around security when interacting with strangers via communication technology. This included worries about sharing personal details (e.g. where they lived), as well as physical identity (e.g. being seen on a webcam). Consequently, contacts were represented using a generic avatar and first names. However, during this study, as the trial progressed and users became familiar with each other, participants expressed desire to have access to some record of information about different people (e.g. interests, where they lived) as well as the ability to see each other via a webcam during calls. More work is needed to see how information can be shared in a graduated way to maintain sense of privacy without hindering social relatedness as people get to know each other.

Fourth, one of the major factors of engagement with technology is perceived relevance. The broadcasts on health were by far the most popular and highly attended than any other type of broadcast. This suggests that informative or educational content, which is perceived as beneficial in itself, acts as an effective channel for encouraging social interaction. This aligns well with the findings of a systematic survey of Loneliness Intervention studies carried out between 1970 and 2002. Nine out of the ten most effective interventions in this survey involved group activities with educational or support input. Participants in the trial suggested that they also enjoyed broadcasts with geographically local information content, news and history [20].

Finally, there was consensus among participants that the system would work best in conjunction with meeting up face to face. Through the trial, primary participants did set up meetings with other participants with whom they found things in common (i.e. went to the same school, similar profession, and so on), and scheduled events that people could attend (i.e. a walk in the park at a certain day and time). Furthermore, at the end of the trial, people expressed desire to meet with other participants and to see their faces (through the possible implementation of a webcam feature). They also suggested that an in-person meeting (possibly for training purposes) before the actual deployment would have helped connectedness among participants. One participant said 'I think there comes a point where you do actually need to meet to become really friendly with somebody. It's a sight barrier, not being able to see their faces. I can't quite say why' (Julia), while another suggested that for the trial to be more successful in 
engaging participants in socialising it 'would [have been] nice if we were met and went walking or to a pub' (Louise). This leads to believe that a technology of this kind would probably work better in conjunction with facilitating some personal, face-to-face interactions. The benefits of using the technology for communicating would be amplified if supplemented with occasional face-to-face contact.

\section{Conclusion}

This paper describes a 10 week home trial with a novel communication system designed to encourage social engagement among older adults who do not know each other in an opportunistic and unobtrusive way. The technology used in the present study emerged through a participatory design process involving older adults to ensure that the design was grounded in an understanding both of user needs and practices $[15,21]$. This home trial was an exploratory study to see how the system was used in the context of real homes outside the laboratory and how it could be improved to encourage social engagement.

The quantitative and qualitative data provided insight into how the system is used in a home context and provided further support in understanding participants' perceptions of the system and how it could be effectively implemented. Despite being a completely new system, participants generally found it easy to use. They reported that it helped social connection and created interactions outside the system; as one participant commented: 'It made me feel as though there was somebody there for me and I was there for somebody' (Louise).

The challenge for such as a system is to maintain social engagement. Findings from the trial suggest that quality of social engagement over time is limited in the absence of a graduated approach to sharing personal information as users become familiar with one another, and even open up to the possibility of meeting face-to-face. The efficacy of the technology would also be enhanced if supplemented with the group meeting before the technology is deployed. The broadcast feature was core to the design as it aimed to provide an opportunity to meet and a topic for conversation. It was observed that this was most successful when the broadcasts included informative or educational content around health, as opposed to less instrumental themes such as comedy, news or stories. This suggests the need to take a dual benefit approach in which opportunities for social interaction co-exist with an opportunity to learn. This study also demonstrates the importance of using both qualitative and quantitative approaches to exploring how technology is used in real contexts to inform the design process and understand how it should be implemented to successfully encourage social engagement. Future directions for this research could explore the novelty effect curve and engagement over time during longer trials; also, intervention studies could be conducted in which loneliness assessments are repeated before and after the deployment to assess potential improvement in people quality of life; finally, engaging with larger numbers of primary and secondary participants would provide statistical power for generalisation to a broader social context.

Acknowledgments Thanks are due to our colleagues, especially Brian Lawlor, Ben Arent, James Brennan, Vanessa Buckley, Julie Doyle, Ronan McDonnell, Blaithin O'Dea, Simon Roberts, Cormac Sheehan, David Singleton, Claire Somerville, Zoran Skrba, Susan Squires, Maurice ten Koppel, Flip van den Berg and Ciaran Wynne. This research was completed as part of a wider programme of research within the TRIL Centre (Technology Research for Independent Living). The TRIL Centre is a multi-disciplinary research centre, bringing together researchers from UCD, TCD, NUIG \& Intel, funded by Intel, IDA Ireland and GE Healthcare. www.trilcentre.org.

\section{References}

1. Pinquart, M., Sorensen, S.: Influences on loneliness in older adults: a metaanalysis. Basic Appl. Soc. Psychol. 23(4), 245-266 (2001)

2. Victor, C., Scambler, S., Bond, J., Bowling, A.: Being alone in later life: loneliness, social isolation and living alone. Rev. Clin. Gerontol. 10, 407-417 (2000)

3. Tijhuis, M.A.R., de Jong-Gierveld, J., Feskens, E.J.M., Kromhout, D.: Changes in and factors related to loneliness in older men: the Zutphen Elderly Study. Age Ageing 28(5), 491-495 (1999)

4. Bowling, A.: The most important thing in life: comparisons between older and younger population age groups by gender. Int. J. Health Sci. 6(4), 169-175 (1995)

5. Sidell, M.: Death, dying and bereavement. In: Bond, J., Coleman, P., Peace, S. (eds.) Ageing in Society: An Introduction to Social Gerontology. Sage Publications, London (1993)

6. Golden, J., Conroy, R.M., Lawlor, B.: Social support network structure in older people: underlying dimensions and association with psychological and physical health. Psychol. Health Med. 14(3), 280-290 (2009)

7. Monk, A.F., Reed, D.J.: Telephone conferences for fun: experimentation in people's homes, pp. 201-214. HOIT, Springer, Chennai, India (2007)

8. King, H.: A telephone reassurance service: a natural support system for the elderly. J. Gerontol. Soc. Work 16(1/2), 159-177 (1991)

9. White, H., McConnell, E., Clipp, E., Bynum, L., Teage, C., Navas, L., Craven, S., Halbrecht, H.: Surfing the net in later life: a review of the literature and pilot study of computer use and quality of life. J. Appl. Gerontol. 18(3), 358-378 (1999)

10. Groves, D.L., Slack, T.: Computers and their application to senior citizens therapy within a nursing home. J. Instruct. Psychol. 21(3), 221-227 (1994)

11. Dickinson, A., Newell, A.F., Smith, M.J., Hill, R.L.: Introducing the Internet to the over-60 s: developing an email system for older novice computer users. Interact. Comput. 17(6), 621-642 (2005)

12. Inoue, M., Suyama, A., Takeuchi, Y., Meshitsuka, S.: Application of a computer based education system for aged persons and issues 
arising during the field test. Comput. Methods Prog. Biomed. 59(1), 55-60 (1999)

13. Czaja, S.J., Guerrier, J.H., Nair, S.N., Landauer, T.K.: Computer communication as an aid to independence for older adults. Behav. Inf. Technol. 12(4), 197-207 (1993)

14. Hawthorn, D.: How universal is good design for older people? In: Proceedings of the ACM Conference on Universal Usability, pp. 38-45. Vancouver, Canada (2003)

15. Wherton, J.P., Prendergast, D.K.: Building bridges: involving older adults in the design of comunication technology to support peer-to-peer social engagement. In: Holzinger, A., Miesenberger, K. (eds.) HCI and Usability for E-Inclusion, pp. 111-134. Springer, Berlin (2009)

16. De Jong Gierveld, J., Van Tilburg, T.: A 6-item scale for overall, emotional and social loneliness: confirmatory tests on survey data. Res. Aging 28(5), 582-598 (2006)
17. Luanaigh, C.O., Lawlor, B.A.: Loneliness and the health of older people. Int. J. Geriatric Psych. 23(12), 1213-1221 (2008)

18. Winer, B.J.: Statistical principles in experimental design. McGraw-Hill, New York (1971)

19. Newell, A., Arnott, J., Carmichael \& Morgan, M.: Methodologies for involving older adults in the design process. In: Stephanidis, C. (ed.) Universal Access in HCI, Part I, pp. 982-989. Springer, Berlin, LNCS 4554 (2007)

20. Cattan, M., White, M., Bond, J., Learmouth, A.: Preventing social isolation and loneliness among older people: a systematic review of health promotion interventions. Ageing Soc. 25, 41-67 (2005)

21. Prendergast, D., Roberts, S.: Practice, systems and technology for seniors: a communication. Univ. Access Inf. Soc. 7(3), 59-61 (2008) 